\title{
Poly(acrylonitrile-co-itaconic acid)-poly(3,4-ethylenedioxythiophene) and poly(3-methoxythiophene) nanoparticles and nanofibres
}

\author{
HAVVA BASKAN ${ }^{1}$, CEM UNSAL ${ }^{1}$, HALE KARAKAS ${ }^{1}$ and A SEZAİ SARAC ${ }^{2, *}$ \\ ${ }^{1}$ Department of Textile Engineering, Istanbul Technical University, Gumussuyu, 34437 Istanbul, Turkey \\ ${ }^{2}$ Nanoscience and Nanoengineering, Polymer Science and Technology, Department of Chemistry, Istanbul Technical \\ University, Maslak, 34469 Istanbul, Turkey \\ *Author for correspondence (sarac@itu.edu.tr)
}

MS received 26 July 2016; accepted 30 November 2016; published online 28 August 2017

\begin{abstract}
This work aimed to produce poly(acrylonitrile-co-itaconic acid) (P(AN-co-IA)) nanocomposites with poly(3,4ethylenedioxythiophene) (PEDOT) and poly(3-methoxythiophene) (PMOT). An anionic surfactant sodium dodecyl benzene sulphonate was used in emulsion polymerization for nanocomposite production. Incorporations of PEDOT and PMOT on the nanoparticles were characterized by scanning electron microscopy (SEM), atomic force microscopy, Fourier transform infrared-attenuated total reflectance spectroscopy and ultra-violet spectroscopy. These nanoparticles were blended with PAN and the blends were electrospun to produce $\mathrm{P}(\mathrm{AN}-\mathrm{co}-\mathrm{IA})-$ polythiophene-derivative-based nanofibres, and the obtained nanofibres were characterized by SEM and energy dispersive spectroscopy. In addition, electrochemical impedance studies conducted on nanofibres showed that PEDOT and PMOT in matrix polymer P(AN-co-IA) exhibited capacitive behaviour comparable to that of ITO-PET. Their capacitive behaviour changed with the amount of electroactive polymer.
\end{abstract}

Keywords. Poly(acrylonitrile-co-itaconic acid); poly(3, 4-ethylenedioxythiophene); poly(3-methoxythiophene); nanoparticle; nanofibre; electrospinning.

\section{Introduction}

There is a great interest for nanocomposite materials due to their advantage of combining the properties of two or more different materials and their good mechanical, electrical and thermal properties [1]. Polyacrylonitrile (PAN), used as a carbon fibre precursor, is one of the mostly used polymers for producing composite materials. As a homopolymer of PAN has strong dipole-dipole interactions, it becomes difficult to obtain fibres during the spinning process [2-4]. In order to overcome the obstacles in spinning, better chain alignment must be achieved and nitrile-nitrile interactions in PAN must be disordered [5]. Therefore some comonomers are used to generate composites with acrylonitrile (AN). Copolymerization of $\mathrm{AN}$ gives the chance of distruption of strong dipole interactions, increasing decomposition temperature and decreasing high melting point of the polymer [6]. Acrylates (methyl acrylate and butyl acrylate), vinyl acetate, ketones, alcohols, acrylic acid and itaconic acid (IA) are among the widely used comonomers [7-12]. Polymerization of AN with vinylacetate was studied by Çetiner et al [6] and it was observed that by addition of vinylacetate to AN, the solubility of the polymer in spinning solutions increased. Sen and Sarac [13] selected IA as a comonomer for the production of poly(acrylonitrile-co-itaconic acid) ( $\mathrm{P}(\mathrm{AN}-\mathrm{co}-\mathrm{IA}))$ polymer, and they showed that using higher IA ratios in polymerization step let to decreased decomposition temperature of the obtained polymer. In a study, Unsal et al [10] copolymerized AN with butylacrylate and pyrrole; they showed that by incorporation of butylacrylate to the system, the mechanical properties of the resulting polymer improved. Copolymerization of P(AN-co-IA)-polypyrrole was achieved by Arman and Sarac [14] via emulsion polymerization for the synthesis of AN and vinylacetate in order to investigate the morphology of the nanocomposites. Bhanu et al [11] used methylacrylate for copolymerizing with $\mathrm{AN}$ and they carried out synthesis and characterization of the produced polymer.

IA is the more preferred acidic comonomer due to its two carboxylic groups, which help in reordering nitrile groups [15]. Devasia et al [15] studied cyclization reaction of P(ANco-IA) and they examined cyclization reaction kinetics. Yu et al [16] investigated thermal properties of AN-IA polymers in oxidative and nonoxidative atmospheres, and showed that IA facilitated oxidation reactions in addition to cyclization reactions. Bajaj et al [17] studied the effect of IA and methylacrylic acid, copolymerized with AN, on the thermooxidative properties, glass transition and melting temperature of PAN. They showed that addition of acidic comomomers to PAN reduces the reaction initiation temperature [17]. Ouyang et al [18] compared the thermal behaviour and 
structure properties of PAN with and without IA comonomer. Tsai and Lin [19] analysed the effect of IA on the exothermic reaction. They concluded that the exothermic peak became more complex and showed several distinct peaks on increasing the molar content of IA, and cyclization reaction rate was largely dependent on the amount of the comonomer in PAN copolymers [19].

Electrically conductive polymers offer good electrical and chemical polymer characteristics [1]. They have a wide range of applications such as electromagnetic shielding, lightemitting diodes, molecular electronics, chemical, biochemical and thermal sensors, etc. However, the characterization and processsibility of conjugated polymers have some limits due to their insoluble and infusible characteristics; therefore, their use in composite form is preferred for better processibility [20]. Among the conjugated polymers, polythiophenes are preferred for their good thermal and chemical stability, thus offering potential for many applications [21]. As in other conjugated polymers, substitutions to the polythiophene can change the processibility of the polymer [20]. In the polythiophene family, poly (3,4-ethylenedioxthiophene) (PEDOT) has drawn interest due to its lower oxidation potential, low band gap, good electrical conductivity and high stability of doped state [22]. Based on these properties, PEDOT has many applications such as biosensors, electrochemical displays, smart windows, antistatic coatings, etc. [23]. Satici and Sarac [24] coated PEDOT on poly(AN-co-vinylacetate)- $\mathrm{Fe}_{2} \mathrm{O}_{3}$ in order to obtain core-shell nanocapsules and they also obtained nanofibres from nanocapsules. Poly(3-methoxythiophene) (PMOT) is another example that has drawn attention because of its high oxidation potential and poor solubility in water. For a long time PMOT had been synthesized only in organic medium [25,26]. PMOT can perform as an electron donor substituent, which decreases the oxidation potential of the polymer due to the existence of oxygen in alkoxy group [27]. For processibility and usage of these conjugated polymers, it would be beneficial to combine them with other polymers.

Electrospinning is a simple and versatile technique to obtain nanofibres that have high surface area to volume ratio, high tensile strength, stiffness and flexibility in surface functionalities. These excellent properties lead them to be used in many industrial applications. The process is also advantageous in terms of cost-effectiveness. A high-voltage supplier, a needle, polymer solution and collector are the components of electrospinning. A polymer solution is prepared and a high voltage is applied to this polymer solution, generating electrical charges in the solution. When the charges reach a critical amount, a Taylor cone forms at the tip of the needle and a fluid jet erupts from the droplet. The fluid jet moves towards the collector, which has lower potential, and it evaporates simultaneously. Polymeric nanofibres can be used in a wide range of applications. For example, they can be implemented in military protective clothing, sensors, wound dressing, drug delivery, tissue engineering and other biological applications, cosmetics, electromagnetic shielding, photovoltaic devices, micro/nano-electronic devices, LCD devices, gas filtration, molecule or air filtration, etc. [28-30].

The present work aims firstly to obtain $\mathrm{P}(\mathrm{AN}-\mathrm{co}-\mathrm{IA})$ nanoparticles and produce nanocomposites of $\mathrm{P}(\mathrm{AN}-\mathrm{co}-$ IA) with PEDOT and with PMOT. By achieving these compositions the obstacles in the usage of homopolymer of PAN and PEDOT and PMOT would be eliminated. Secondly, it aims to prepare nanofibres from the resulting nanoparticles. The effects of the conjugated polymers in the composites were demonstrated by Fourier transform infrared-attenuated total reflectance (FTIR-ATR), ultraviolet (UV)-visible spectroscopy, atomic force microscopy (AFM) and scanning electron microscopy (SEM) characterizations of the produced nanoparticles. In addition, the obtained nanofibres were also characterizated by SEM, energy dispersive spectroscopy (EDS) and electrochemical impedance study (EIS). The obtained copolymers and nanofibres can be used in a variety of applications such as nanocoatings for textile industry, gas sensors, biosensors, etc., with further improvements where the electroactivity is required.

\section{Experimental}

\subsection{Materials}

$\mathrm{AN}(>99.5 \%)$ was provided by Aksa Acrylic Chemistry Company. IA $(>99.5 \%), 3,4$-ethylenedioxythiophene (EDOT, $>97 \%$ ), 3-methoxythiophene (MOT, >98\%), sodium dodecyl benzene sulphonate (SDBS) and PAN $\left(M_{\mathrm{w}}=150,000\right)$ were purchased from Sigma-Aldrich. Ammonium per sulphate (APS), N,N dimethylformamide (DMF) and ethanol were all Merck reagents. All these reagents were used without further purification.

\subsection{Synthesis of $P(A N-$ co-IA $)$ copolymers}

$\mathrm{P}(\mathrm{AN}-\mathrm{co}-\mathrm{IA})$ latex was produced by emulsion polymerization using SDBS as surfactant and APS as initiator. During the polymerization, $0.1 \mathrm{~mol}$ monomer mixture in total was used and the feed ratio of AN to IA was selected as 99 mol\%; $200 \mathrm{ml}$ of polymerization medium was copolymerized in a three-necked flask. In the first stage, the surfactant SDBS was dissolved in $150 \mathrm{ml}$ distilled water and stirred for $15 \mathrm{~min}$. The ratio of surfactant to total monomer was determined to be $20 \mathrm{wt} \%$. The monomers IA and AN were incorporated into the solution and stirred for approximately $30 \mathrm{~min}$. Until this process, $5.3831 \mathrm{~g}$ monomer was consumed. After that, the polymerization temperature was set at $70^{\circ} \mathrm{C}$ and the polymerization medium was heated to this temperature in the flask, while being stirred with reflux. When the temperature reached $70^{\circ} \mathrm{C}$, the initiator APS, which had been dissolved in $10 \mathrm{ml}$ distilled water, was fed into the system. The ratio of APS to monomers in the feed was $15 \mathrm{wt} \%$. The reaction was continued for 


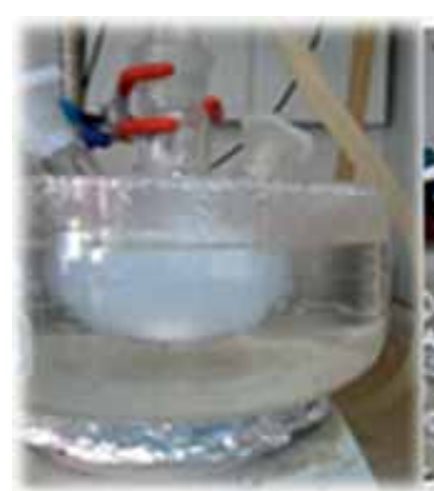

(a)

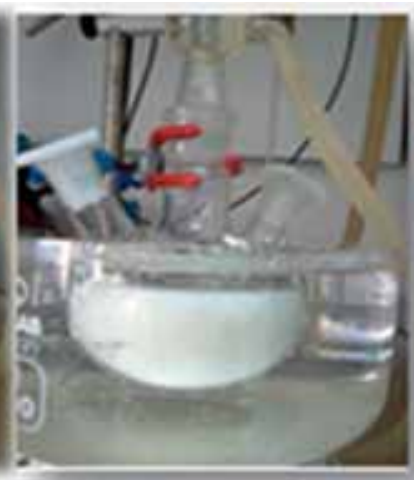

(b)

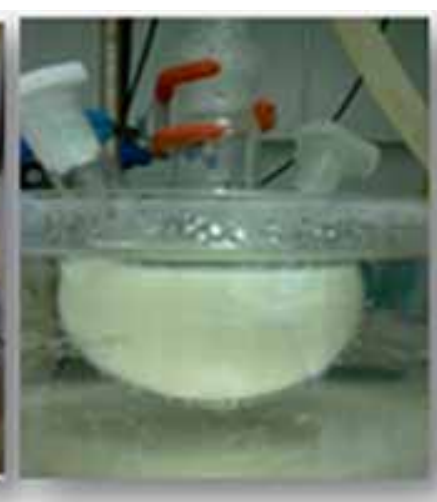

(c)

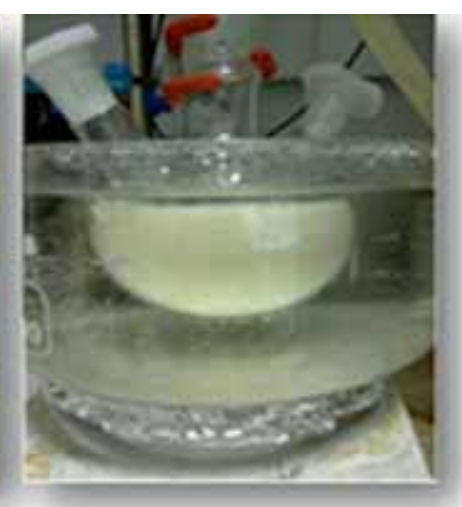

(d)

Figure 1. Emulsion latex at (a) the beginning of the copolymerization; (b) $1 \mathrm{~h}$ after the copolymerization; (c) $2 \mathrm{~h}$ after the copolymerization and (d) $3 \mathrm{~h}$ after the copolymerization.

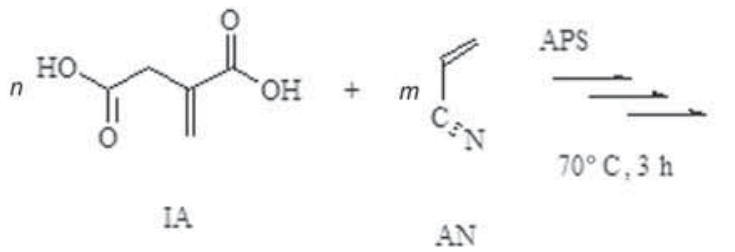

IA
AN

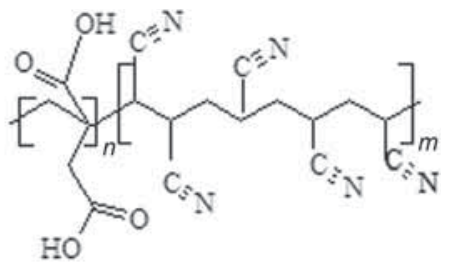

P(AN-co-IA)

Figure 2. Reaction scheme of $\mathrm{P}(\mathrm{AN}-\mathrm{co}-\mathrm{IA})$.

$3 \mathrm{~h}$; the colour change for each hour in the polymerization medium has been shown in figure 1. At the end of $3 \mathrm{~h}$, copolymerization was stopped by closing the heater. Finally, P(AN-co-IA) milky-fluid latex was obtained. The chemical reaction between AN and IA has been shown in figure 2.

EDOT and MOT were used for coating P(AN-co-IA) latex nanoparticles by an in-situ polymerization technique. At the end of the copolymerization of $\mathrm{P}(\mathrm{AN}-\mathrm{co}-\mathrm{IA})$, the temperature was reduced by cooling from $70^{\circ} \mathrm{C}$ to room temperature, while the latex was being stirred continuously. Then the $200 \mathrm{ml}$ synthesized copolymer solution was divided into 10 containers, each containing $20 \mathrm{ml}$ of solution. With no initiator addition into the containers, EDOT and MOT were incorporated into the emulsion medium seperately and the reaction was maintained for $72 \mathrm{~h}$. Three different amounts of EDOT, 83.3, 125 and $166.6 \mu \mathrm{l}$, and three different amounts of MOT, 77.6, 116.4 and $155.2 \mu \mathrm{l}$, were added into the containers. Figure 3 shows a schematic drawing of coated PEDOT on the matrix polymer ( $\mathrm{P}(\mathrm{AN}-\mathrm{co}-\mathrm{IA}))$ at the end of in-situ polymerization. At the end of $72 \mathrm{~h}$, obtained nanoparticles were precipitated using ethanol. For eliminating impurities encountered during the polymerization process, filtration was applied to the precipitated solution and precipitated nanoparticles were washed with distilled water and ethanol five times. Finally the nanoparticles were dried at $60^{\circ} \mathrm{C}$ in a vacuum oven.

\subsection{Electrospinning of nanocomposite structures}

In the electrospinning process, three different amounts of P(AN-co-IA)-PEDOT and P(AN-co-IA)-PMOT composite nanoparticles were blended with PAN in DMF for tuning the viscosity of the electrospinning solutions due to the inadequacy of the viscosity of produced nanocomposite solutions. In the blend, the ratio of the produced copolymer to PAN was $20 \%$ in volume. PAN solutions were stirred at $400 \mathrm{rpm}$ for $2 \mathrm{~h}$ and simultaneously solutions containing produced copolymers were stirred at $400 \mathrm{rpm}$ for $2 \mathrm{~h}$. They were then blended and stirred again for $2 \mathrm{~h}$. The solutions were then loaded into a $2.5 \mathrm{ml}$ syringe and pumped. The electrospinning parameters were selected as $5 \mathrm{wt} \%$ polymer concentration in solution, $15 \mathrm{kV}$ voltage, $1.0 \mathrm{ml} \mathrm{h}^{-1}$ feed ratio and $10 \mathrm{~cm}$ distance from needle tip to collector. The nanofibres were collected on the grounded collector covered with aluminium foil.

\subsection{Spin coating}

Precipitated and dried composite nanoparticles and matrix polymer nanoparticle were treated with DMF to obtain a polymer solution concentration of $5 \mathrm{wt} \%$. Later, the treated solutions were dripped on pure glasses and spin coating was applied for $1 \mathrm{~min}$ with a spin-coater device. Finally, spincoated glasses were examined in detail by AFM in order to see the morphology of the nanoparticles. 


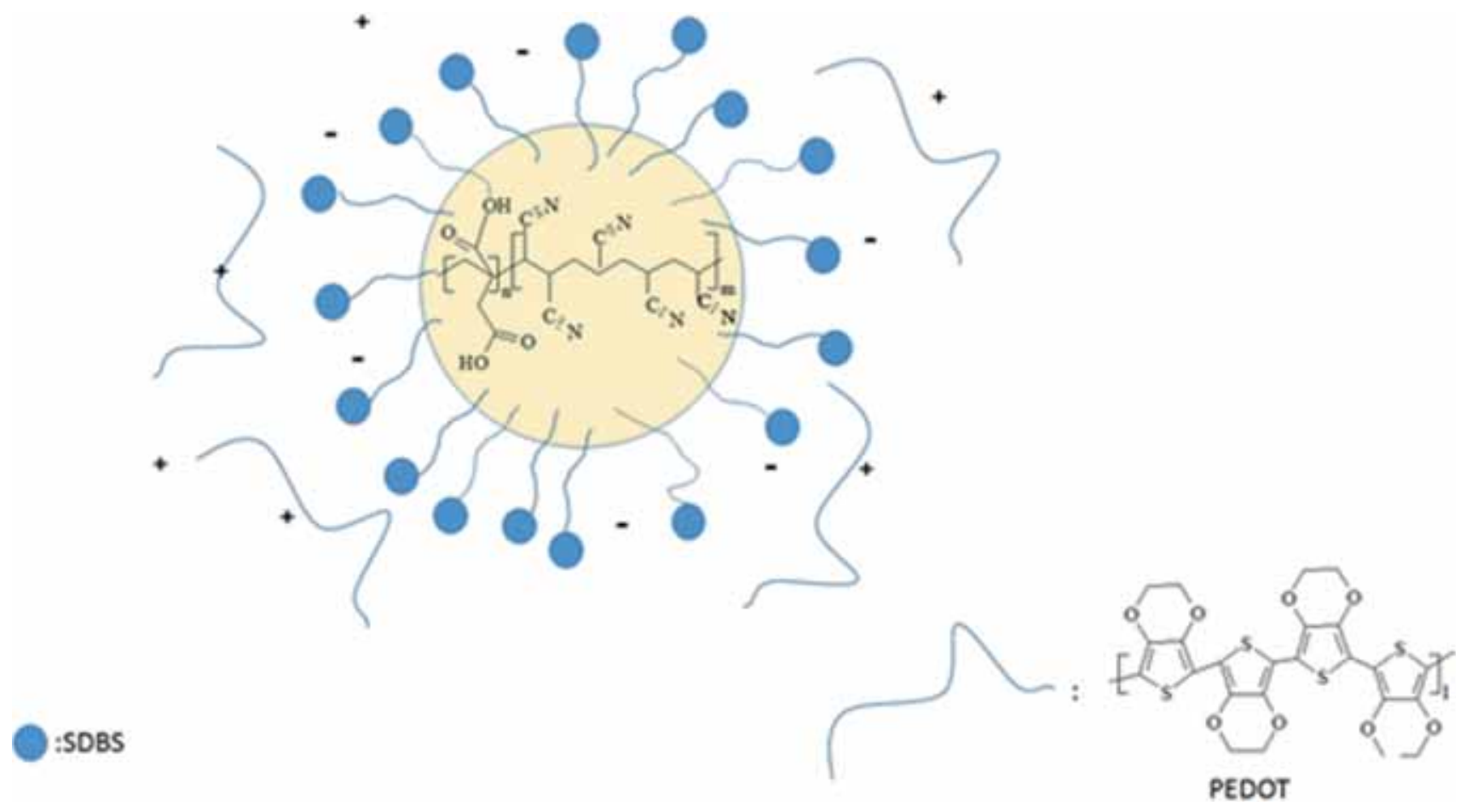

Figure 3. Reaction scheme of $\mathrm{P}(\mathrm{AN}-\mathrm{co}-\mathrm{IA})-\mathrm{PEDOT}$.

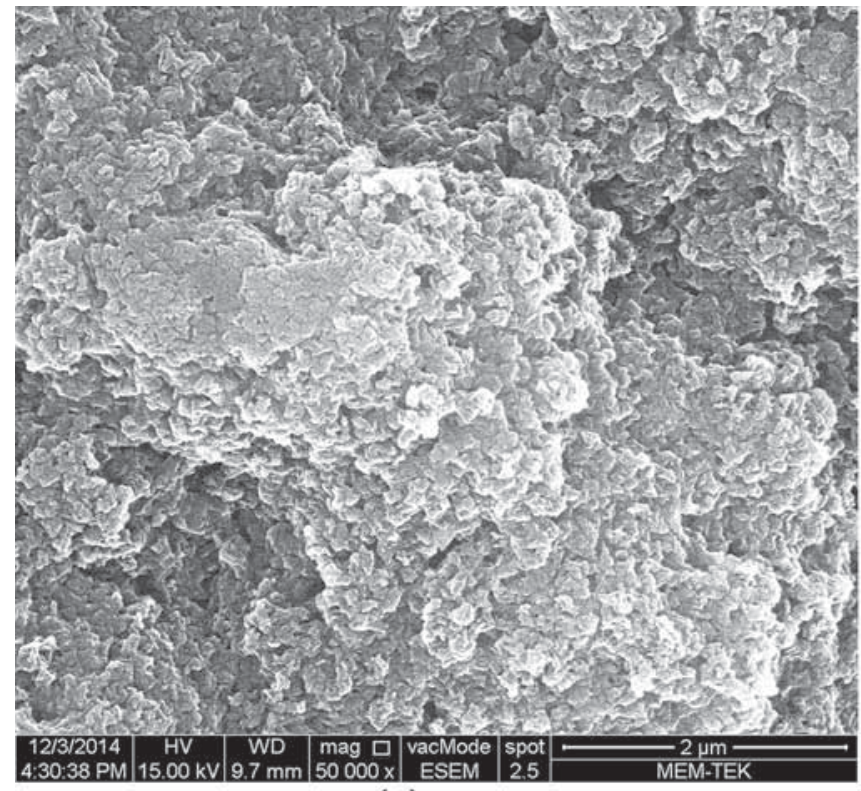

(a)

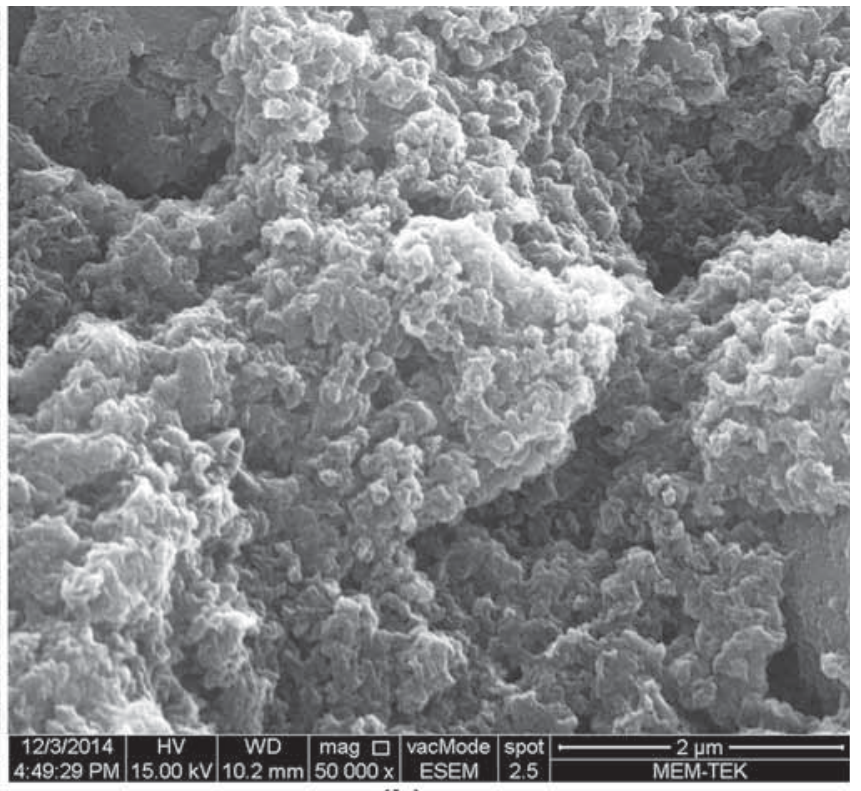

(b)

Figure 4. SEM image of (a) $\mathrm{P}(\mathrm{AN}-\mathrm{co}-\mathrm{IA})$ (matrix polymer) and (b) $\mathrm{P}(\mathrm{AN}-\mathrm{co}-\mathrm{IA})$-PEDOT nanoparticles containing $166.6 \mu \mathrm{l}$ EDOT.

\subsection{Characterization}

The latex obtained from emulsion polymerization and the solutions comprising PEDOT and PMOT were diluted to the optimum value and the absorbance changes were tested with a UV-visible spectrometer (Perkin-Elmer, Lambda 45).
The FTIR-ATR analysis of precipitated and dried copolymers were carried out using an FTIR-ATR reflectance spectrophotometer (Perkin-Elmer, Spectrum One, with a universal ATR attachment with a diamond and ZnSe crystal). The precipitated and dried nanoparticles were examined by scanning electron microscopy (SEM, FEI Quanta FEC 250). 


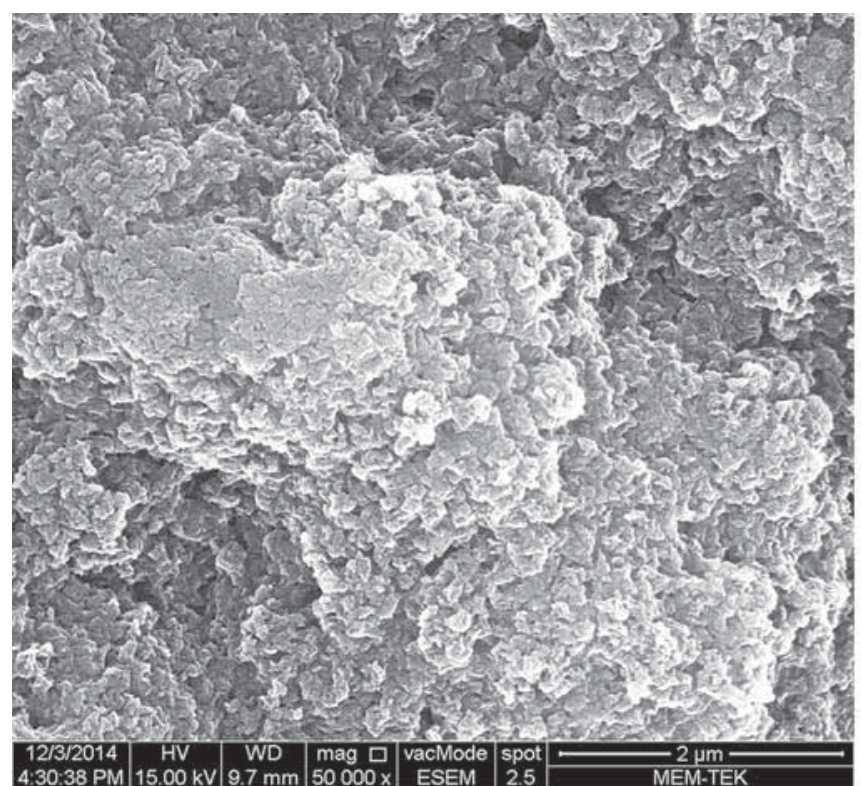

(a)

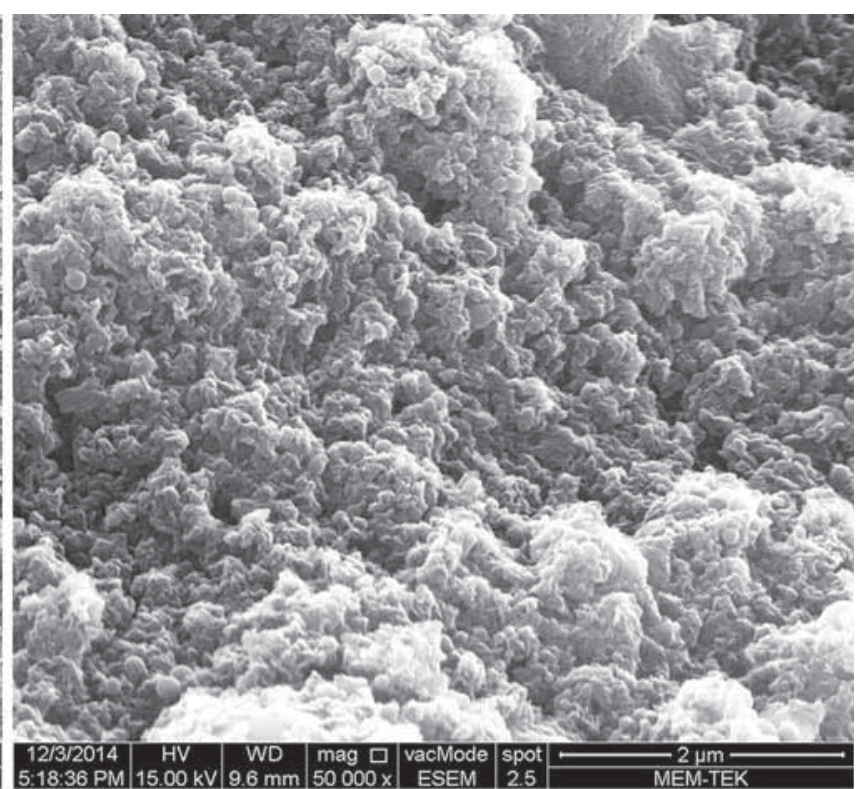

(b)

Figure 5. SEM image of (a) $\mathrm{P}(\mathrm{AN}-\mathrm{co}-\mathrm{IA})$ (matrix polymer) and (b) $\mathrm{P}(\mathrm{AN}-\mathrm{co}-\mathrm{IA})-\mathrm{PMOT}$ nanoparticles containing $155.2 \mu \mathrm{MOT}$.

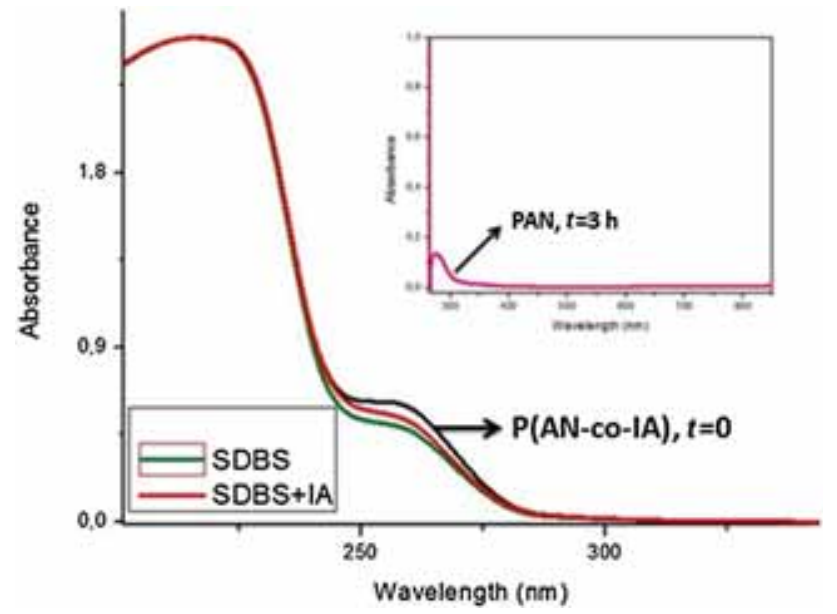

Figure 6. UV-visible spectroscopy of SDBS, SDBS+IA, P(ANco-IA) at $t=0$ and homopolymer of PAN at $t=3 \mathrm{~h}$.

Surface morphology characterizations of the spin-coated samples were observed by AFM (Nanosurf Easy Scan 2). In order to obtain nanofibres, an electrospinning set-up (NE500 Model, New Era Pump System Inc., USA) and a DC power supplier (ES 50 Model, Gamma High Voltage Inc., USA) were used. The viscosity of the electrospinning solutions was measured in the range of $0.3-10000 \mathrm{mPa}$ s using an 'Vibro Viscometer SV-10'. SEM and EDS characterizations of nanofibres were performed using a FEI Quanta FEC 250 instrument. Electrochemical measurements (EIS measurements) were performed with a Princeton Applied Research (PAR) Parstat 2263 potentiostat by scanning in the frequency range $10 \mathrm{mHz}-100 \mathrm{kHz}$ with an applied AC signal amplitude of $10 \mathrm{mV}$ using a Power Sine.

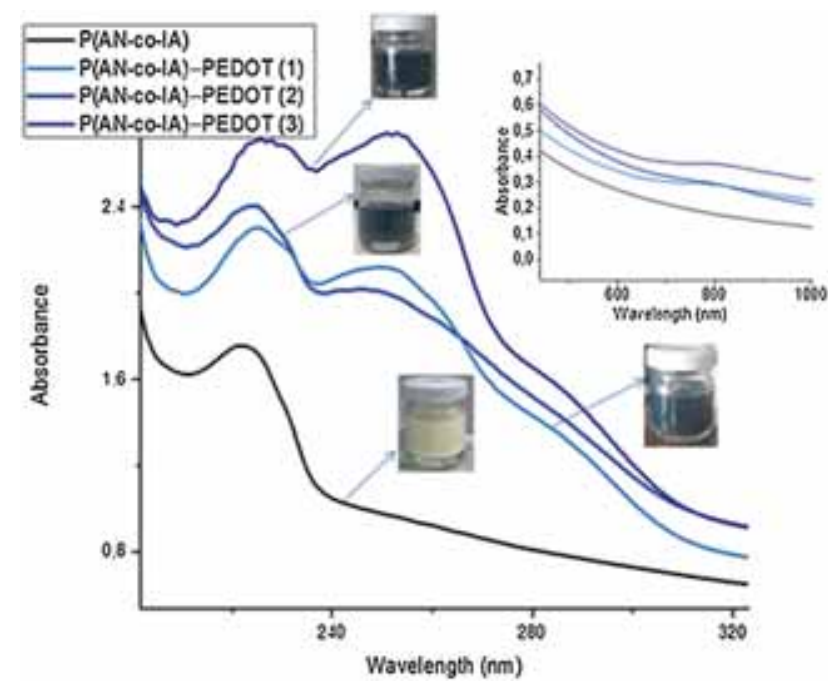

Figure 7. UV-visible spectroscopy of $\mathrm{P}(\mathrm{AN}-\mathrm{co}-\mathrm{IA})$, (1) $\mathrm{P}(\mathrm{AN}-\mathrm{co}-$ IA)-PEDOT (83.3 $\mu$ l), (2) P(AN-co-IA)-PEDOT (125 $\mu \mathrm{l})$ and (3) P(AN-co-IA)-PEDOT (166.6 $\mu \mathrm{l})$.

\section{Results and discussion}

\subsection{SEM analysis of nanoparticles}

Surface morphology of the matrix polymer and nanoparticles containing PEDOT was observed and compared by SEM characterization. Figure 4 a shows an image of the matrix polymer and plates in the structure of the copolymer can be clearly seen. The surface roughness of matrix polymer is less when compared with the nanoparticles containing PEDOT. Unlike the matrix polymer, the porosity of the structures is obviously 
high since porosity depends on the presence of electroactive polymer in the structure. Morphology of the nanoparticles exhibits difference, i.e., in figure $4 \mathrm{~b}$, a cauliflower structure is seen in the morphology rather than plates. Similar SEM images for PEDOT containing nanoparticles are also present in the literature. For example, in the study conducted by Park, PEDOT containing nanoparticles characterized by SEM images were defined as microspheres with cauliflower morphology [31]. In another study, some grains containing PEDOT were generated and their SEM images showed a 'cauliflower-like' structure [32].

Figure 5a shows the matrix polymer and $5 \mathrm{~b}$ shows the nanocomposite polymer structure of $\mathrm{P}(\mathrm{AN}-\mathrm{co}-\mathrm{IA})-\mathrm{PMOT}$. As in the $\mathrm{P}$ (AN-co-IA)-PEDOT composite nanoparticles, the morphology of the $\mathrm{P}(\mathrm{AN}-\mathrm{co}-\mathrm{IA})-\mathrm{PMOT}$ composite nanoparticles is like a cauliflower. For the nanoparticles of matrix polymer, the growth is in the same plate, and they do not prefer to grow in $3 \mathrm{D}$ as in the case of nanoparticles containing PMOT.

Thus, both PEDOT and PMOT are embedded into the matrix polymer, forming a homogeneous nanocomposite structure as $\mathrm{P}(\mathrm{AN}-\mathrm{co}-\mathrm{IA})-\mathrm{PEDOT}$ and $\mathrm{P}(\mathrm{AN}-\mathrm{co}-\mathrm{IA})-\mathrm{PMOT}$.

\subsection{UV-visible and FTIR-ATR spectroscopy}

Absorbance values of surfactant SDBS, SDBS+IA and P(ANco-IA) at $t=0$ have been shown in figure 6. In this UV-visible spectroscopy experiment, the absorbance values were 0.5 , 0.54 and 0.60 for SDBS, which is the surfactant, SDBS+IA and $\mathrm{P}(\mathrm{AN}-\mathrm{co}-\mathrm{IA})$ at $t=0$, respectively. In addition, the absorbance value of PAN homopolymer has also been shown for comparing the reactivity ratios (inset of figure 6).

Dilution ratio of the solution was $1 / 500$; finally, when the absorbance values of the peaks are compared, it can be seen that ratio of absorbance of $\mathrm{P}(\mathrm{AN}-\mathrm{co}-\mathrm{IA})$ to $\mathrm{PAN}$ is found to be 1.46. This means that polymerization of $\mathrm{AN}$ and IA at the end of $3 \mathrm{~h}$ is 1.46 times faster than AN. Monomer reactivity ratios of acrylonitrile/itaconic acid was found to be approximately 6. In the literature, studies focussed on the reactivity ratio of AN to IA can be found and the values mentioned in these references are close to the value 6 [33]. In another study by Devasia et al [15], it was mentioned that 'IA radicals have very little reactivity towards their own monomer unit; AN radical has a greater preference to the IA unit than its own monomer'.

Figure 7 shows that absorbance increases with an increase in PEDOT amount. From the figure, it can be seen that near $250 \mathrm{~nm}$ (in the UV region) there is a peak due to the PEDOT. For three different amounts of PEDOT, a broad peak was observed between the values 1.8 and 2.8. Furthermore, near $800 \mathrm{~nm}$ (in the visible region), peaks related to the PEDOT can be seen approximately in the absorbance range of $0.3-0.5$.

Figure 8 shows the absorbance values of $\mathrm{P}(\mathrm{AN}-\mathrm{co}-\mathrm{IA})$ and $\mathrm{P}(\mathrm{AN}$-co-IA)-PMOT compositions with three different amounts. Near $255 \mathrm{~nm}$, a clear peak and a broad band are seen with an increase in the amount of PMOT. The absorbance values varied between 1.3 and 1.7.

If the PEDOT containing nanoparticles are compared to each other, the reactivity ratio values of 1.14 and 1.17 can be evaluated from figure 8 if the lower value of PEDOT is taken as the baseline. If this procedure is performed for PMOT containing nanoparticles, monomer reactivity ratios of 1.15 and 1.23 can be evaluated.

Before the FTIR characterization the produced nanoparticles were precipitated and dried, and their yield was evaluated. Table 1 summarizes the yield values of the copolymers. It can

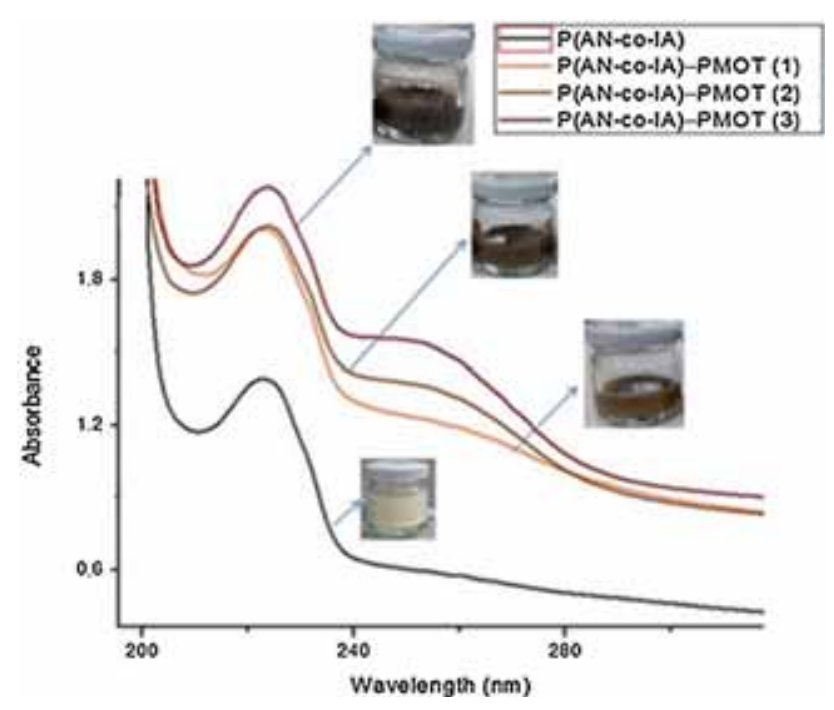

Figure 8. UV-visible spectroscopy of $\mathrm{P}(\mathrm{AN}-\mathrm{co}-\mathrm{IA})$, (1) $\mathrm{P}(\mathrm{AN}-\mathrm{co}-$ IA)-PMOT (77.6 $\mu \mathrm{l}),(2) \mathrm{P}(\mathrm{AN}-\mathrm{co}-\mathrm{IA})-\mathrm{PMOT}(116.4 \mu \mathrm{l})$ and (3) $\mathrm{P}(\mathrm{AN}-\mathrm{co}-\mathrm{IA})-\mathrm{PMOT}(155.2 \mu \mathrm{l})$.

Table 1. Yield of the produced copolymers.

\begin{tabular}{|c|c|c|c|}
\hline Copolymer & $\begin{array}{l}\text { Initial } \\
\text { mass }(\mathrm{g})\end{array}$ & $\begin{array}{l}\text { Final mass } \\
\text { (after drying) (g) }\end{array}$ & Efficieny (\%) \\
\hline P(AN-co-IA) & 0.6 & 0.456 & 76 \\
\hline $\begin{array}{l}\text { P(AN-co- IA })- \\
\text { PEDOT }(83.3 \mu l)\end{array}$ & 0.6 & 0.438 & 73 \\
\hline $\begin{array}{l}\mathrm{P}(\mathrm{AN}-\mathrm{co}-\mathrm{IA})- \\
\text { PEDOT } \\
(125 \mu \mathrm{l})\end{array}$ & 0.6 & 0.462 & 77 \\
\hline $\begin{array}{l}\text { P(AN-co-IA })- \\
\text { PEDOT } \\
(166.6 \mu \mathrm{l})\end{array}$ & 0.6 & 0.444 & 74 \\
\hline $\begin{array}{l}\text { P(AN-co-IA })- \\
\text { PMOT } \\
(77.6 \mu l)\end{array}$ & 0.6 & 0.420 & 70 \\
\hline $\begin{array}{l}\text { P(AN-co-IA)- } \\
\text { PMOT } \\
(116.4 \mu \mathrm{l})\end{array}$ & 0.6 & 0.444 & 74 \\
\hline $\begin{array}{l}\text { P(AN-co-IA })- \\
\text { PMOT } \\
(155.2 \mu \mathrm{l})\end{array}$ & 0.6 & 0.456 & 76 \\
\hline
\end{tabular}



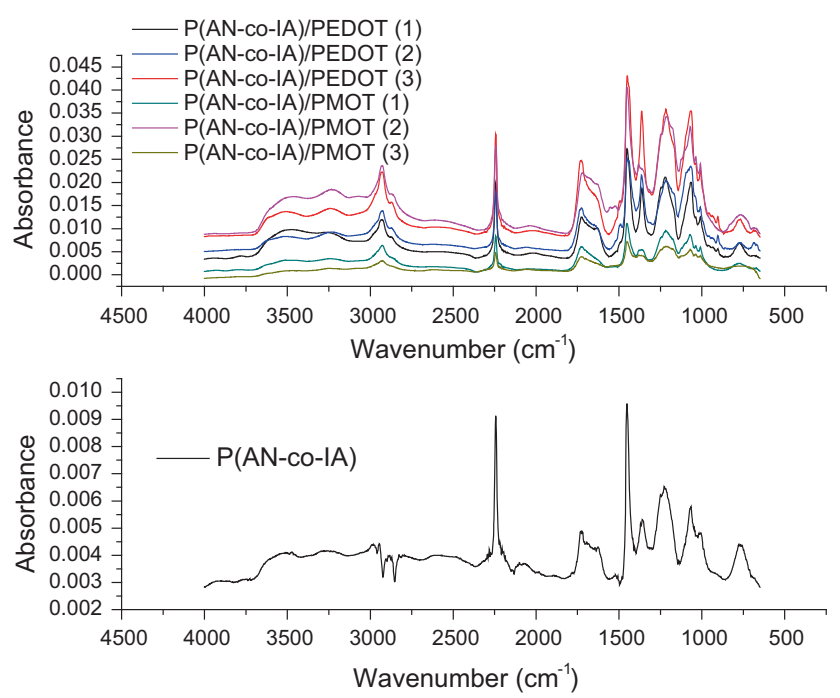

Figure 9. FTIR-ATR spectroscopy of $\mathrm{P}(\mathrm{AN}-\mathrm{co}-\mathrm{IA})$, (1) $\mathrm{P}(\mathrm{AN}-\mathrm{co}-$ IA)-PMOT (77.6 $\mu \mathrm{l})$, (2) P(AN-co-IA)-PMOT (116.4 $\mu \mathrm{l})$ and (3) P(AN-co-IA)-PMOT (155.2 $\mu$ l), (1) P(AN-co-IA)-PEDOT (83.3 $\mu \mathrm{l})$, (2) P(AN-co-IA)-PEDOT (125 $\mu$ l) and (3) P(AN-co-IA)PEDOT $(166.6 \mu \mathrm{l})$.

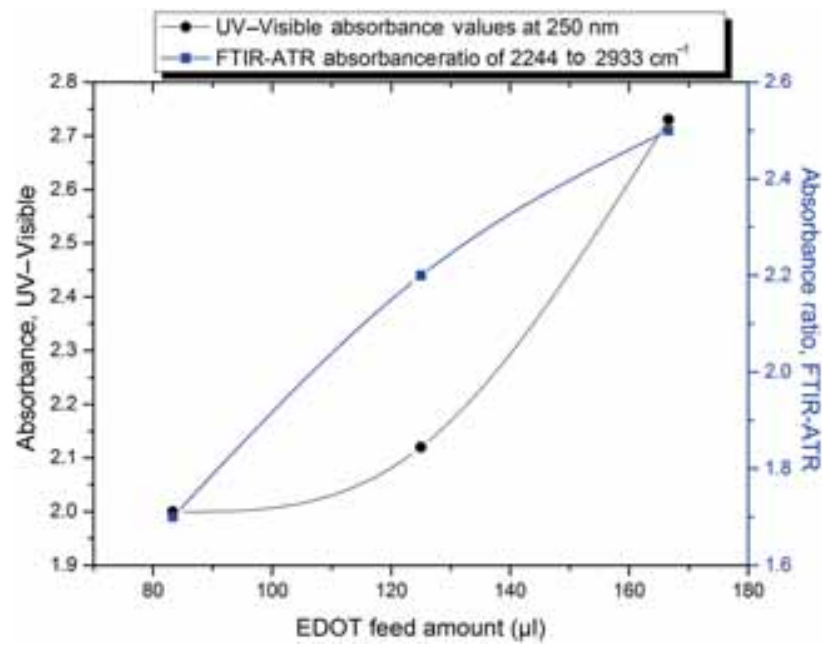

Figure 10. Absorbance of FTIR-ATR and UV-visible spectrums vs. EDOT feed amount in $\mu$ l.

be said that the efficiency of the polymerization processes ranges from 70 to $76 \%$.

Figure 9 displays the FTIR-ATR spectroscopy results of $\mathrm{P}$ (AN-co-IA) and P(AN-co-IA) with PEDOT and with PMOT. Increasing amounts of PEDOT and PMOT can be inferred from the graph. PAN shows its characteristic absorption peaks at 1453 and $2244 \mathrm{~cm}^{-1}$, corresponding to $\mathrm{C} \equiv \mathrm{N}$ stretching and bending vibration of $-\mathrm{CH}$, respectively. The aliphatic $\mathrm{CH}_{2}$ stretching vibration of carboxylic acid shows its peak at $2933 \mathrm{~cm}^{-1}$ and the carbonyl stretching vibration at $1733 \mathrm{~cm}^{-1}$. For composites including PEDOT, peaks were observed at 1218,1063, 1008 and $771 \mathrm{~cm}^{-1}$ wavenumbers as

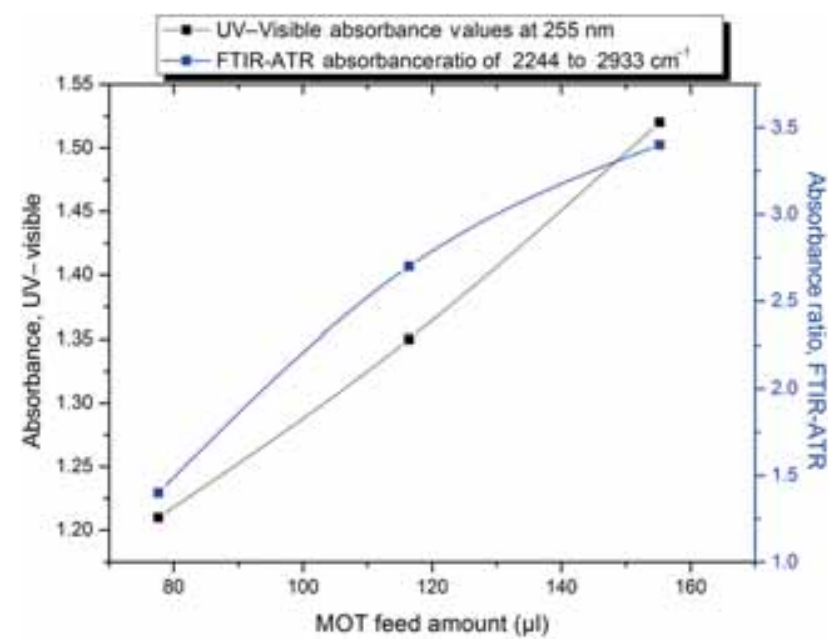

Figure 11. Absorbance of FTIR-ATR and UV-visible spectrums $v s$. MOT feed amount in $\mu$ l.

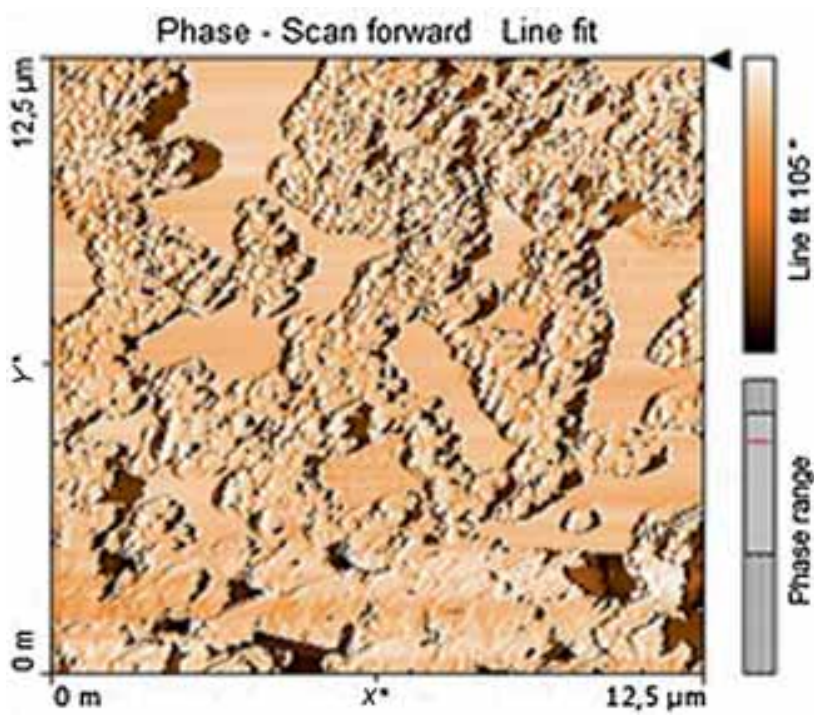

Figure 12. AFM image of smooth glass.

opposed to composites including PMOT. They correspond to ethylenedioxy ring and the $\mathrm{C}-\mathrm{S}$ bond. For PMOT composites, peaks at 1221, 1068 and $774 \mathrm{~cm}^{-1}$ wavenumbers correspond to stretching vibration of $\mathrm{C}-\mathrm{C}$ bond and $\alpha-\alpha$ coupling, respectively.

The relationship between FTIR-ATR and UV-visible spectroscopy results is presented in figures 10 and 11 . The characteristic peak of PAN at $2244 \mathrm{~cm}^{-1}$, bending vibration of $-\mathrm{CH}$ and $2933 \mathrm{~cm}^{-1}$ aliphatic $\mathrm{CH}_{2}$ stretching were used in order to calculate the increase in PEDOT and PMOT amounts. Moreover, results were correlated with UV-visible spetroscopy results, which showed the increase in absorbance at $250 \mathrm{~nm}$ for PEDOT and $255 \mathrm{~nm}$ for PMOT. 


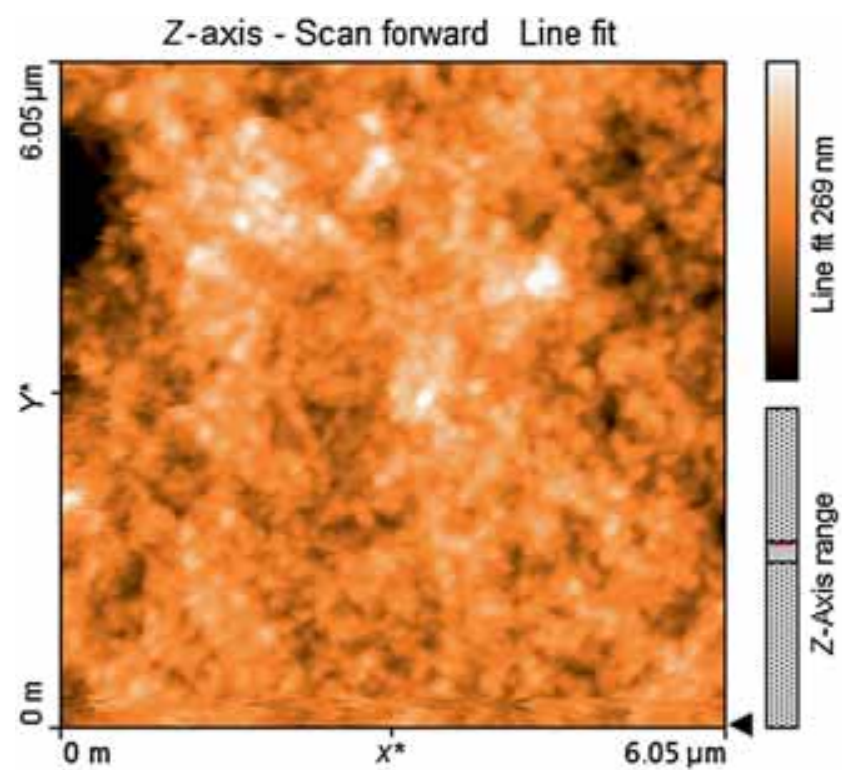

(a)

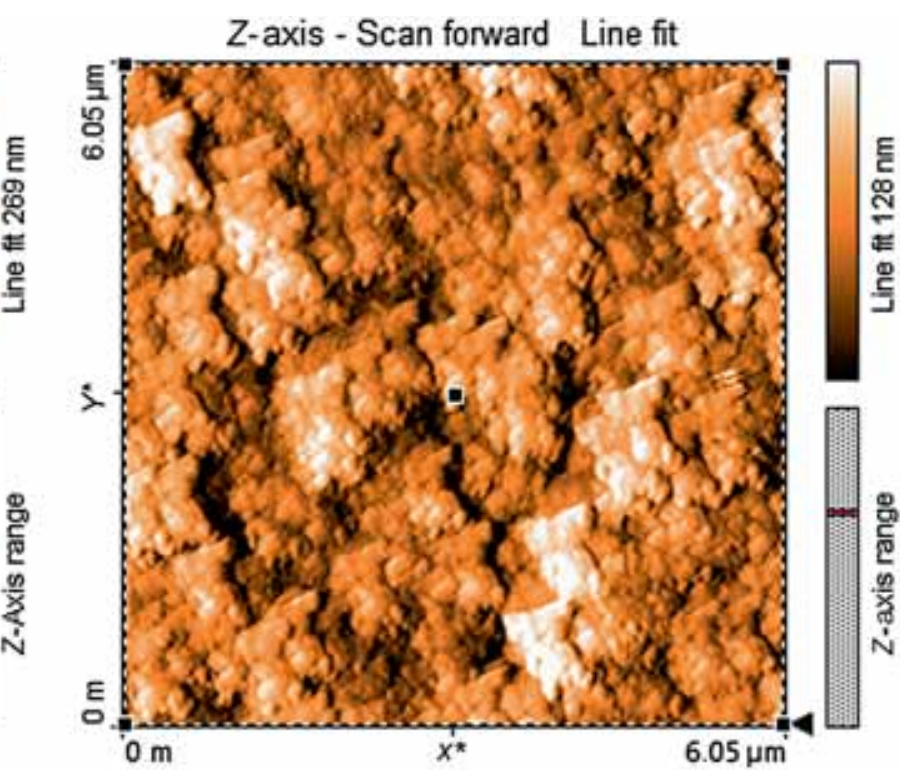

(b)

Figure 13. AFM image of (a) $\mathrm{P}(\mathrm{AN}-\mathrm{co}-\mathrm{IA})$ (matrix polymer) and (b) $\mathrm{P}(\mathrm{AN}-\mathrm{co}-\mathrm{IA})-\mathrm{PEDOT}$ nanoparticles containing $166.6 \mu \mathrm{l}$ EDOT.

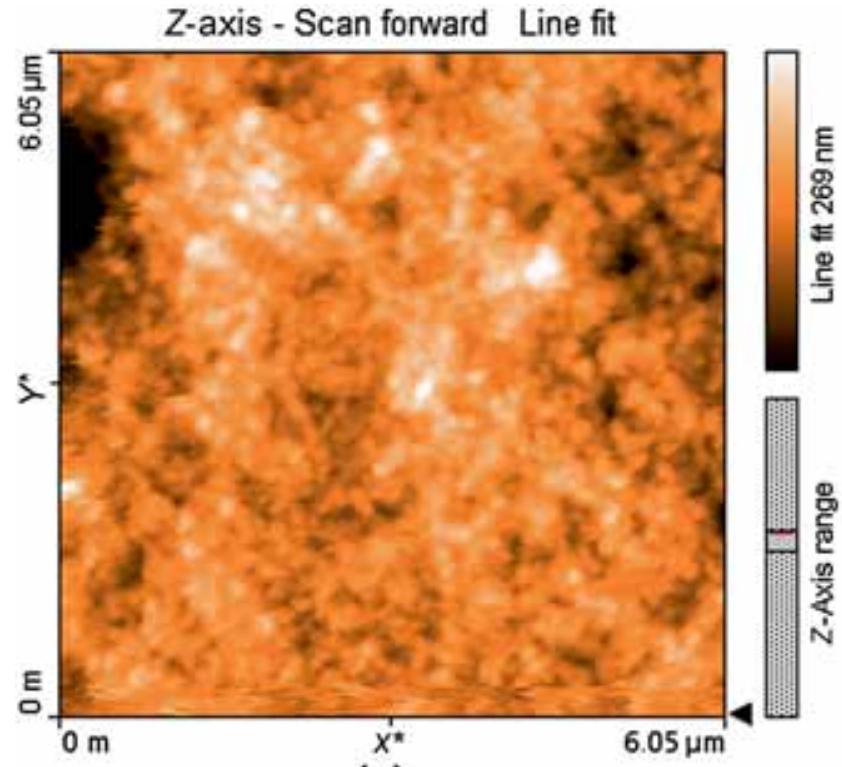

(a)

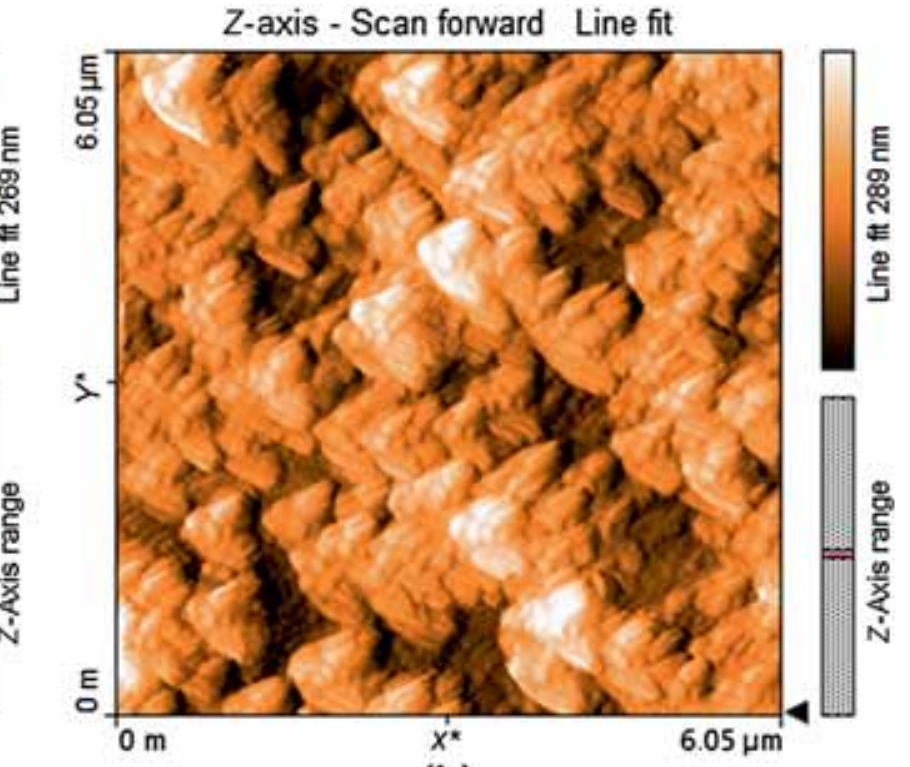

(b)

Figure 14. AFM image of (a) $\mathrm{P}(\mathrm{AN}-\mathrm{co}-\mathrm{IA})$ (matrix polymer) and (b) $\mathrm{P}(\mathrm{AN}-\mathrm{co}-\mathrm{IA})-\mathrm{PMOT}$ nanoparticles containing $155.2 \mu \mathrm{l}$ MOT.

\subsection{AFM analysis of nanoparticles}

Figure 12 shows an AFM image of smooth glass. Surface roughness of the smooth glass was measured to be $6.05 \mathrm{~nm}$.

Surface roughness value of the $\mathrm{P}(\mathrm{AN}-\mathrm{co}-\mathrm{IA})$ film shown in figure 13 is $15.58 \mathrm{~nm}$, which is higher than the surface roughness value of smooth glass. P(AN-co-IA)-PEDOT nanoparticles have also been shown in figure 13. When the film is examined in detail, it can be seen that particles are homogeneously distributed over the film. AFM images reveal that there are some particles in the solution prepared for spin coating and the particle size increases when EDOT is added during the polymerization. The growth of the nanoparticles also changes when it extends to 3D. Furthermore, the surface roughness values increase, respectively, to $15.87,16.08$ and $17.39 \mathrm{~nm}$ for $83.3 \mu \mathrm{l}$ P(AN-co-IA)-PEDOT, $125 \mu \mathrm{l}$ P(AN-co-IA)-PEDOT and 166.6 $\mu \mathrm{l}$ P(AN-co-IA)PEDOT. 


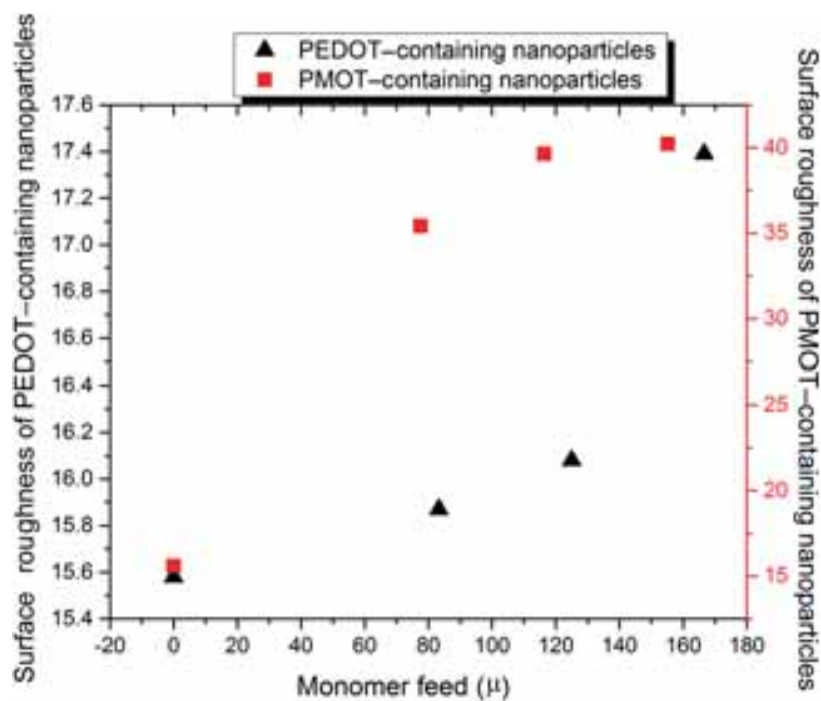

Figure 15. Relationship of the surface roughness of films $v s$. added EDOT and MOT amounts.

AFM images of $\mathrm{P}(\mathrm{AN}-\mathrm{co}-\mathrm{IA})$ and $\mathrm{P}(\mathrm{AN}-\mathrm{co}-\mathrm{IA})-\mathrm{PMOT}$ films are presented in figure 14. The common feature in all AFM images of P(AN-co-IA)-PMOT films is the regularly aligned interesting triangular shapes. The existence of the shapes may depend on the structure of MOT. The 3D growth of the nanoparticles, which was mentioned when the SEM results were discussed, can be seen in AFM images more clearly. Tsegaye et al [34] characterized PMOT-PEDOT films and their AFM image of the PMOT nanoparticles also displayed a triangular shape structure. This growth depends on the electroactive monomer type and triangular shapes are seen for the monomer MOT. In addition, the roughness values were measured to be 35.42, 39.64 and 40.22 for 77.6, 116.4 and $155.2 \mu \mathrm{l}$ MOT, respectively.

Figure 15 shows increase of the surface roughness values with an increase in EDOT and MOT amounts added during in-situ polymerization.

It can be obviously seen that with the addition of electroactive monomers into the polymerization medium, surface roughness increases. The increase of surface roughness is sharper in nanocomposites containing PEDOT when compared with nanocomposites containing PMOT.

\subsection{Morphology of electrospun nanofibres}

In the section on the experimental part, it was mentioned that in order to optimize the viscosity, produced nanoparticles were blended with PAN. Figure 16 shows the relationship among viscosity values of PAN, produced nanocomposite structures and blend of PAN and produced nanocomposite structures. While the viscosity of PAN was $48.8 \mathrm{mPa} \mathrm{s}$, viscosity of produced nanoparticles containing electroactive monomer (P(AN-co-IA)-PEDOT and P(AN-co-IA)-PMOT)

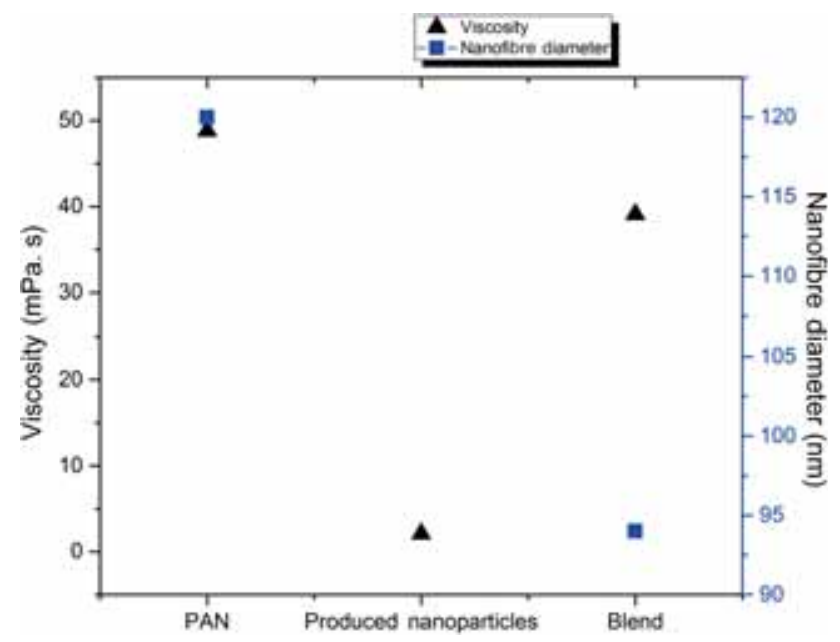

Figure 16. Relationship between viscosity values of PAN, produced nanoparticle, blend and nanofibre diameters.

was measured to be $2.09 \mathrm{mPa}$ s. It can be observed that the viscosity of the produced nanoparticles was too low to spin nanofibres by means of electrospinning; hence, blending with PAN was decided. After blending, viscosity increased to 39.1 $\mathrm{mPa}$ s, which can be seen clearly in figure 16 .

Figure 17 displays SEM images of the electrospun nanofibres along with graphics of their diameter distribution. The average diameter of 100 randomly selected nanofibres was determined using a unique program, Image J, from SEM images of 50,000 magnification. The diameter of the nanofibres varied in the range of $94-180 \mathrm{~nm}$. When compared with PAN or PAN-P(AN-co-IA) nanofibres, nanofibres containing PEDOT and PMOT were observed to have lower nanofibre diameters due to interaction of PEDOT and PMOT with the matrix polymer, $\mathrm{P}(\mathrm{AN}-\mathrm{co}-\mathrm{IA})$. In addition, diameters of the nanofibres decreased with increase in PEDOT and PMOT amount, resulting in smooth and fine nanofibres. For comparison, it can be said that the nanofibres obtained from PAN-P(AN-co-IA)-PMOT blends are smoother than the nanofibres obtained from PAN-P(AN-co-IA)-PEDOT blends. Figure 17 shows the electrospun nanofibres obtained.

Standard deviation from SEM images of the obtained nanofibres was also calculated by taking into account 100 random nanofibre samples and standard deviation results have been given along with average nanofibre diameters in table 2 .

It can be observed that the distribution of the diameters of the electrospun nanofibres displays a narrow range (90-180 $\mathrm{nm}$ ) and the standard deviations are below $40 \%$.

Nanofibre diameter distribution has been presented in figure 18. In the figure, black points show nanofibres containing PEDOT and red points show nanofibres containing PMOT. Nanofibres without any electroactive monomer had larger diameters compared with the nanofibres containing electroactive monomer. Electroactive monomer also led to a decrease in the fibre diameter for both PEDOT- and PMOTcontaining nanofibres, as shown in figure 18 . 


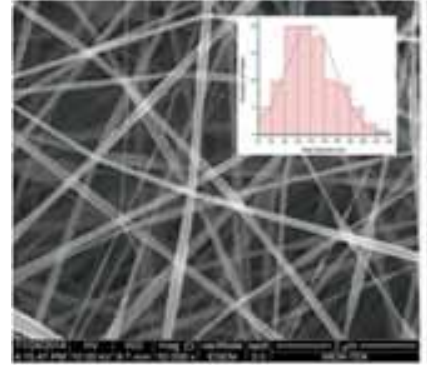

(a)

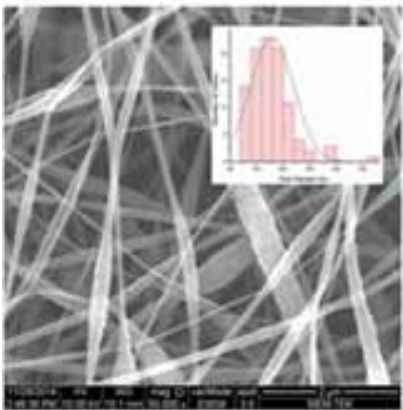

(c)

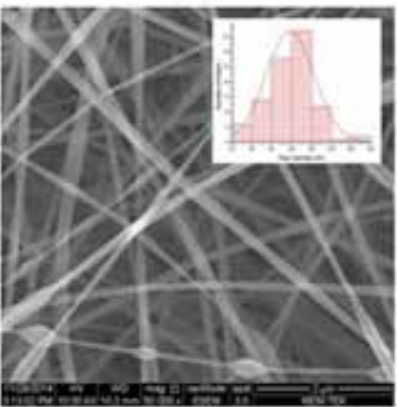

(e)

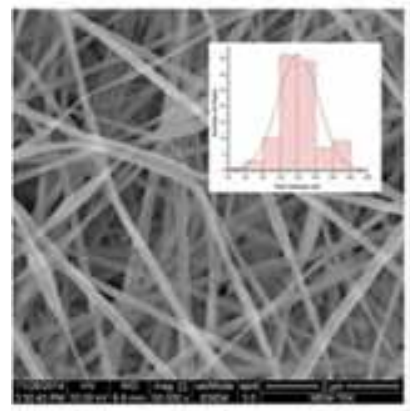

(g)

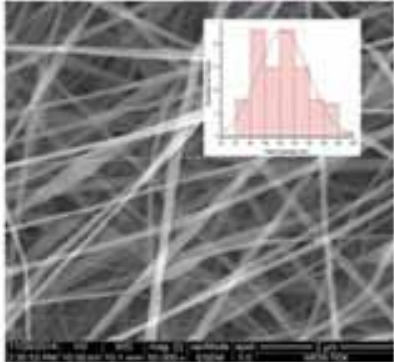

(b)

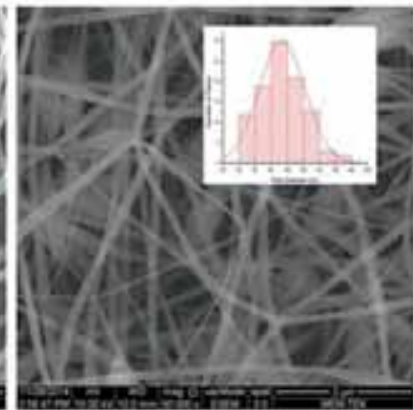

(d)

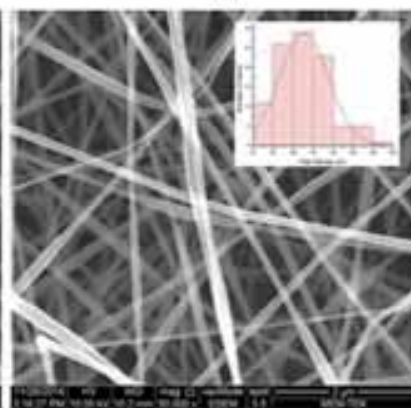

in

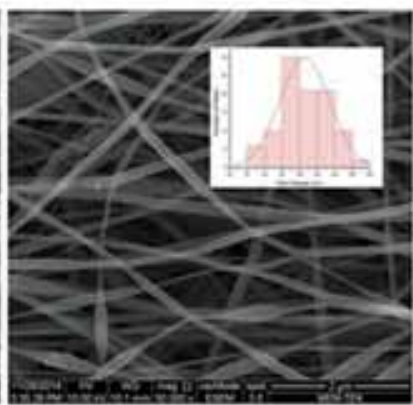

(b)
Figure 17. SEM images of nanofibres: (a) PAN, (b) PAN$\mathrm{P}(\mathrm{AN}-\mathrm{co}-\mathrm{IA}), \quad$ (c) $\mathrm{PAN}-\mathrm{P}(\mathrm{AN}-\mathrm{CO}-\mathrm{IA})-\mathrm{PEDOT} \quad(83.3 \mu \mathrm{l})$, (d) PAN-P(AN-Co-IA)-PEDOT $(125 \mu \mathrm{l})$, (e) PAN-P(ANco-IA)-PEDOT $(166.6 \mu \mathrm{l}), \quad$ (f) $\quad$ PAN-P(AN-co-IA)-PMOT $(77.6 \mu \mathrm{l}), \quad(\mathrm{g}) \quad \mathrm{PAN}-\mathrm{P}(\mathrm{AN}-\mathrm{co}-\mathrm{IA})-\mathrm{PMOT} \quad(116.4 \mu \mathrm{l}) \quad$ and (h) PAN-P(AN-co-IA)-PMOT (155.2 $\mu$ l).

\section{$3.5 E D S$}

Since the viscosity of the produced nanoparticles was too low for electrospinning, it was decided to blend them with PAN. Hence, it was necessary to prove that this blend
Table 2. List of the nanofibre diameters and standard deviation.

\begin{tabular}{lcc}
\hline Blend & $\begin{array}{c}\text { Average fibre } \\
\text { diameter (nm) }\end{array}$ & $\begin{array}{c}\text { Standard } \\
\text { deviation }(\%)\end{array}$ \\
\hline PAN & 120 & 38.00 \\
PAN-P(AN-co-IA) & 124 & 36.39 \\
PAN-P(AN-co-IA)-PEDOT & 180 & 40.62 \\
$\quad(83.3 \mu \mathrm{l})$ & & \\
PAN-P(AN-co-IA)-PEDOT & 94 & 28.94 \\
$\quad(125 \mu \mathrm{l})$ & & \\
PAN-P(AN-co-IA)-PEDOT & 99 & 24.24 \\
$\quad(166.6 \mu \mathrm{l})$ & & \\
PAN-P(AN-co-IA)-PMOT & 110 & 24.85 \\
$\quad(77.6 \mu \mathrm{l})$ & & \\
PAN-P(AN-co-IA)-PMOT & 120 & \\
$\quad(116.4 \mu \mathrm{l})$ & & \\
PAN-P(AN-co-IA)-PMOT & 105 & \\
$\quad(155.2 \mu \mathrm{l})$ & & \\
\hline
\end{tabular}

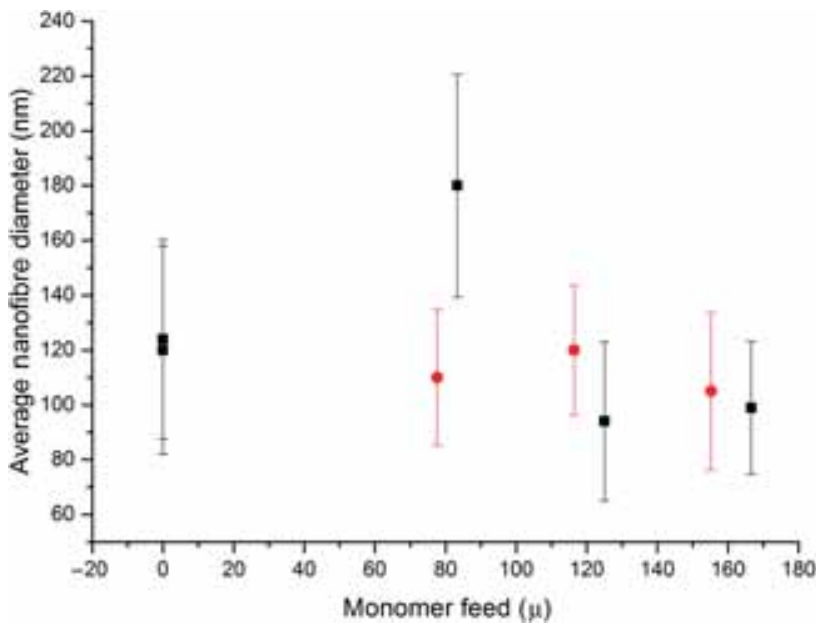

Figure 18. Distribution of the nanofibre diameters.

contained electroactive monomer and that it had been successfully mixed. Also, it was found necessary to investigate the amount of sulphur atoms coming from thiophenes. As a result, EDS characterization, which uses X-ray spectrum obtained by bombarding a focussed beam of electrons, was conducted for chemical analysis of produced samples. All elements can be examined by EDS, by which the amount of the elements in the sample can be easily measured.

Table 3 summarizes EDS results of nanofibres in atomic percentages. Existence of sulphur atoms in the nanofibres confirms that electrospinning solutions, which were obtained by blending PAN with $\mathrm{P}(\mathrm{AN}-\mathrm{co}-\mathrm{IA}), \mathrm{P}(\mathrm{AN}-\mathrm{co}-\mathrm{IA})-\mathrm{PEDOT}$ and $\mathrm{P}(\mathrm{AN}$-coIA)-PMOT, were successfully prepared and mixing was well achieved. In addition, atomic percentages of sulphur increased on increasing the amount of feed EDOT and MOT in the course of in-situ polymerization. 
Table 3. EDS results of the blends.

\begin{tabular}{|c|c|c|c|c|}
\hline Blends & $\mathrm{C}(\%)$ & $\mathrm{N}(\%)$ & $\mathrm{O}(\%)$ & $\mathrm{S}(\%)$ \\
\hline PAN-P(AN-co-IA) & 69.52 & 19.88 & 10.60 & 0 \\
\hline $\begin{array}{c}\text { PAN-P(AN-co-IA)- } \\
\text { PEDOT }(83.3 \mu l)\end{array}$ & 58.78 & 27.48 & 13.51 & 0.23 \\
\hline $\begin{array}{c}\text { PAN-P(AN-co-IA)- } \\
\text { PEDOT }(125 \mu \mathrm{l})\end{array}$ & 55.92 & 32.24 & 11.55 & 0.30 \\
\hline $\begin{array}{l}\text { PAN-P(AN-co-IA)- } \\
\text { PEDOT (166.6 } \mu \mathrm{l})\end{array}$ & 59.97 & 29.77 & 09.92 & 0.34 \\
\hline $\begin{array}{l}\text { PAN-P(AN-co-IA)- } \\
\text { PMOT }(77.6 \mu l)\end{array}$ & 61.55 & 29.17 & 08.91 & 0.37 \\
\hline $\begin{array}{c}\text { PAN-P(AN-co-IA)- } \\
\text { PMOT (116.6 } \mu \mathrm{l})\end{array}$ & 52.09 & 27.78 & 19.76 & 0.38 \\
\hline $\begin{array}{l}\text { PAN-P(AN-co-IA)- } \\
\text { PMOT }(77.6 \mu l)\end{array}$ & 76.27 & 18.97 & 04.15 & 0.61 \\
\hline
\end{tabular}

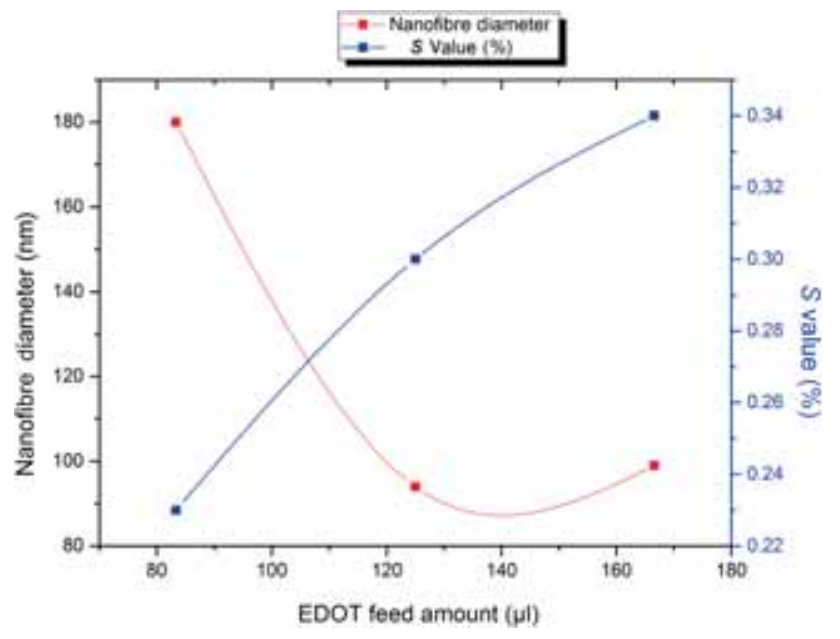

Figure 19. Relationship between nanofibre diameter and sulphur $(\%)$ value results $v$. EDOT feed amount $(\mu \mathrm{l})$.

Figure 19 shows the relationship between nanofibre diameter and sulphur value (\%) results vs. EDOT feed amount $(\mu l)$ during the polymerization process. This figure shows that with an increase in EDOT feed amount, electrospun nanofibre diameter decreases from 180 to $99 \mathrm{~nm}$ where $S(\%)$ value increases from 0.23 to 0.34 .

As in figure 19, figure 20 shows the relationship between obtained nanofibre diameter and sulphur (\%) value $v s$. MOT feed amount $(\mu 1)$ during the polymerization process. In this figure, with an increase in MOT feed amount, a change occurred in the increasing trend of nanofibre diameter; it decreased to $105 \mathrm{~nm}$, while $S(\%)$ value increased from 0.37 to 0.61 .

From the figures, it can be said that addition of EDOT and MOT, to the polymerization medium leads to decrease in electrospun nanofibre diameter.

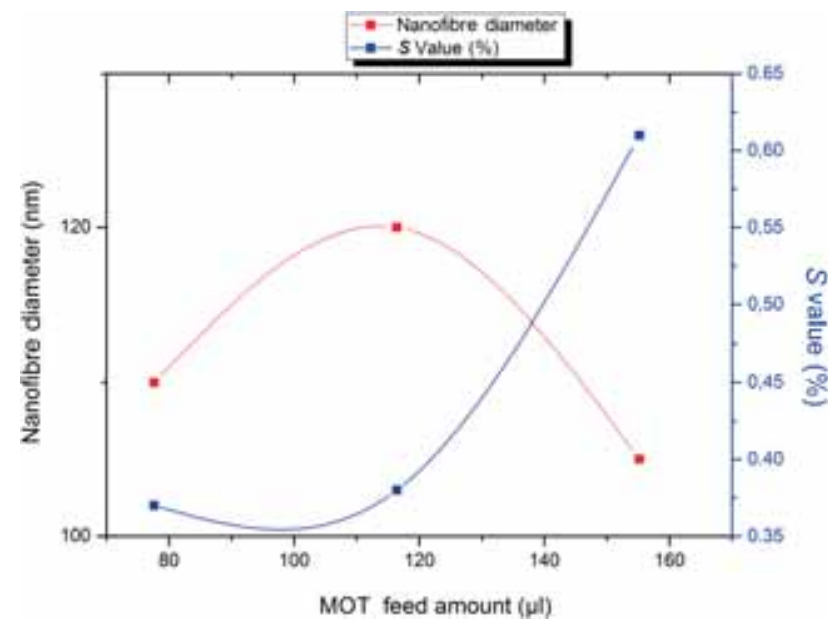

Figure 20. Relationship between nanofibre diameter and sulphur (\%) value results $v s$. MOT feed amount $(\mu \mathrm{l})$.

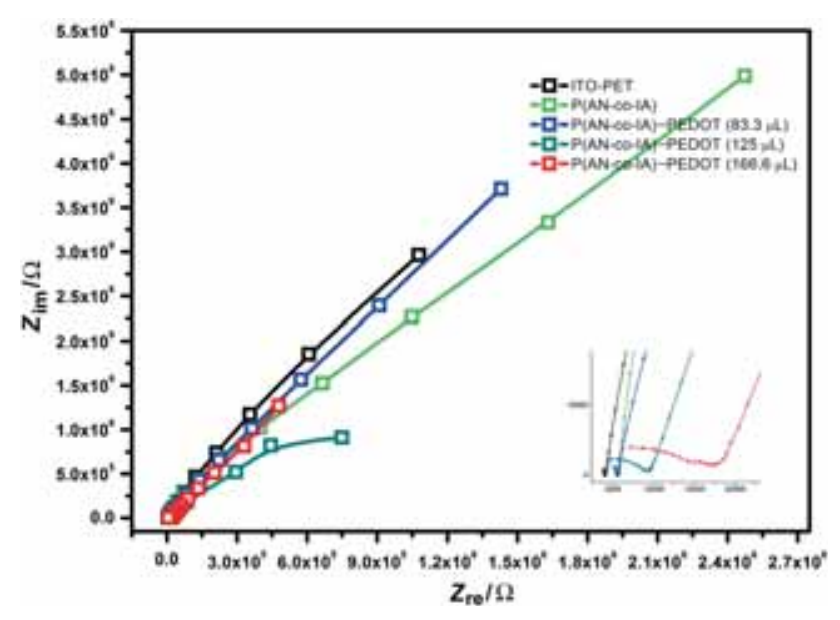

Figure 21. Nyquist graphic of the nanofibres of nanoparticles containing PEDOT blended with PAN.

\subsection{Electrochemical measurements}

EIS measurements were performed in $\mathrm{LiCIO}_{4}(0.1 \mathrm{M})$ solution using a potentiostat 2263 Electrochemical Analyser (Princeton Applied Research, Tennessee, USA) in the frequency range of $0.01 \mathrm{~Hz}-100 \mathrm{kHz}$. EIS was carried out using a three-electrode system where platinum and silver were used as counter and reference electrodes.

EIS data were used to obtain information on the reaction occuring at the electrode-electrolyte interface. Electroactivity of the produced nanofibres was measured in order to demonstrate and compare the capacitive behaviours of the nanofibres produced from the nanoparticles including PEDOT and PMOT. For this reason, Nyquist plots of the nanofibres were used.

Figure 21 shows Nyquist graphic of the nanofibres of nanoparticles containing PEDOT blended with PAN and 


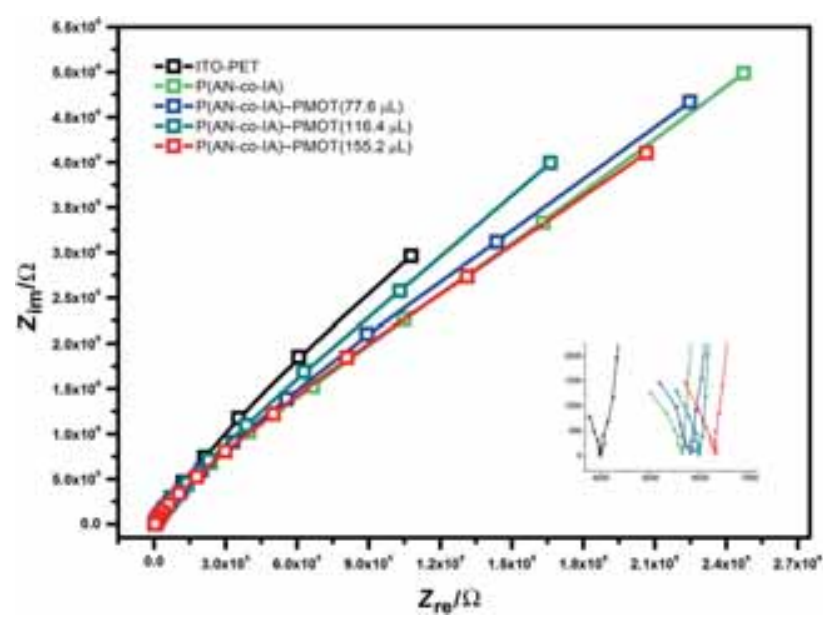

Figure 22. Nyquist graphic of the nanofibres of nanoparticles containing PMOT blended with PAN.

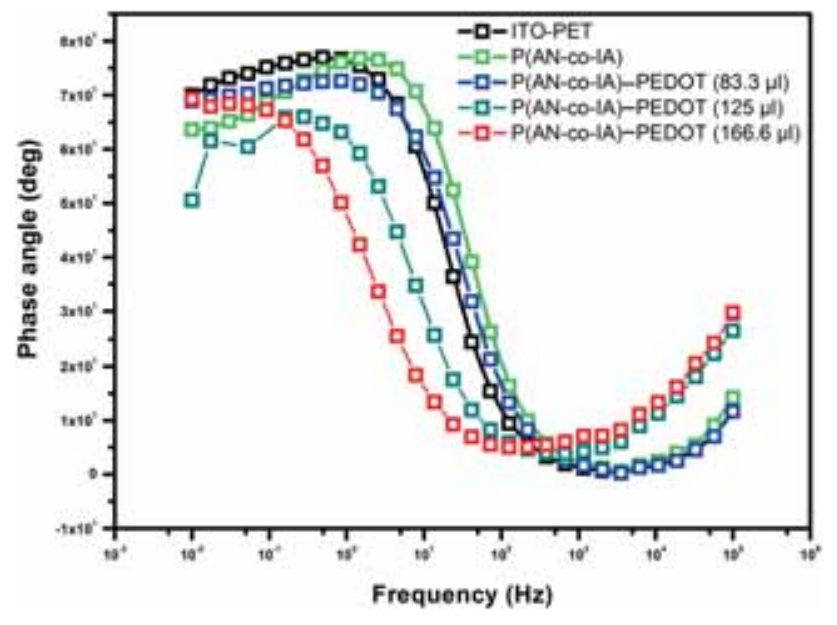

Figure 23. Bode-phase graphic of the nanofibres of nanoparticles containing PEDOT blended with PAN.

figure 22 shows the Nyquist graphic of nanofibres of nanoparticles containing PMOT blended with PAN. When figures 21 and 22 are examined, it can be seen that nanofibres containing PEDOT and PMOT are more capacitive compared with the matrix polymer $\mathrm{P}(\mathrm{AN}-\mathrm{co}-\mathrm{IA})$. As the amount of EDOT or MOT increases, capacitance of the nanofibres also increases. In addition, the nanofibres are also more resistant when the plots are closer to the imaginary part, that is, $y$-axis of the graphic.

Figures 23 and 24 show the Bode-phase graphic of the nanofibres of nanoparticles containing PEDOT and PMOT blended with PAN, respectively. Based on the phase angle observed in the diagram, the capacitive behaviour of the nanofibres increased by an increase in EDOT or MOT amount fed in in-situ polymerization, although the same trend was not observed for the conductivity change by the change of electroactive polymer content (as shown in impedance values $v s$. frequency plots, figures 25 and 26).

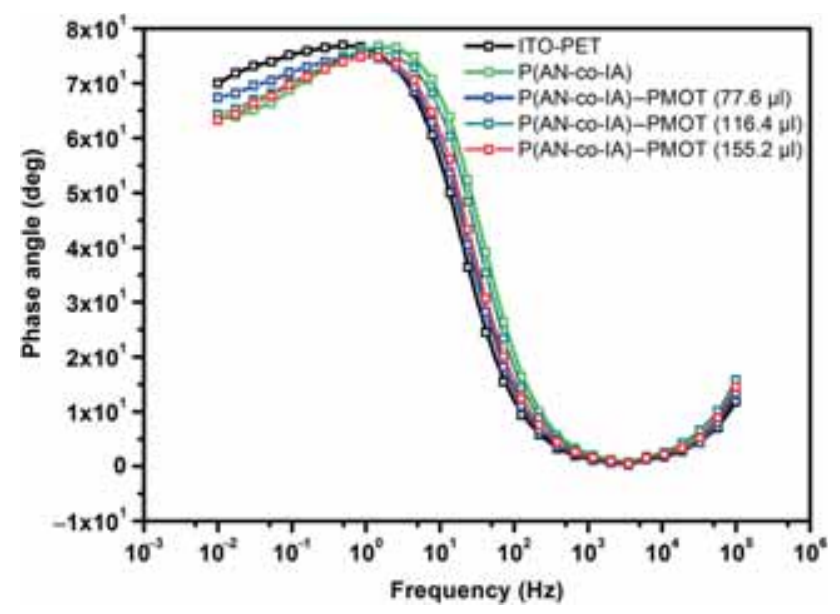

Figure 24. Bode-phase graphic of the nanofibres of nanoparticles containing PMOT blended with PAN.

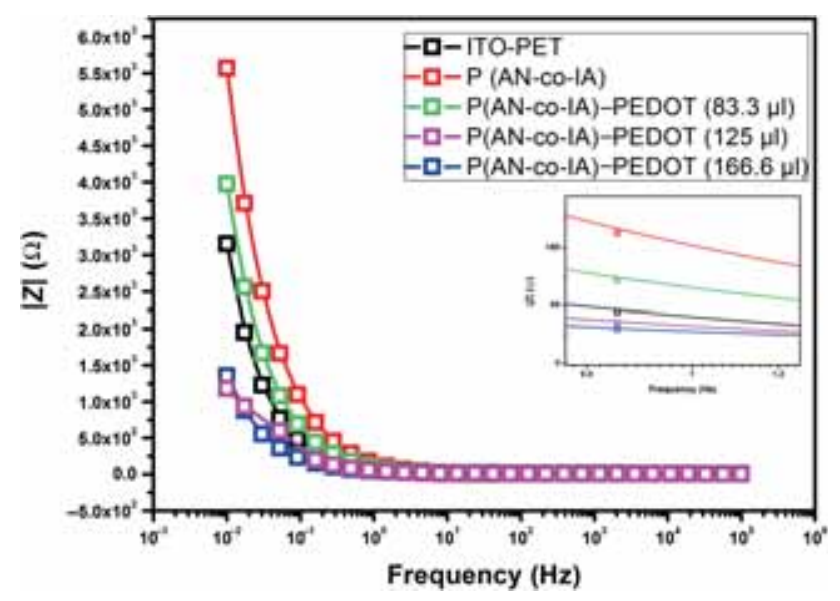

Figure 25. Bode-magnitude graphic of the nanofibres of nanoparticles containing PEDOT blended with PAN.

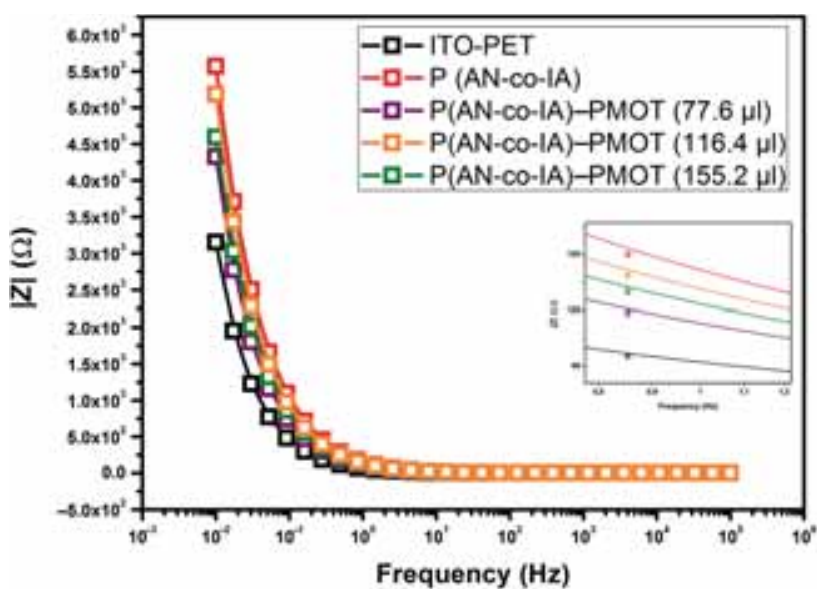

Figure 26. Bode-magnitude graphic of the nanofibres of nanoparticles containing PMOT blended with PAN. 


\section{Conclusion}

In this study, copolymerization of AN and IA was achieved by emulsion polymerization and emulsion latex was obtained. After that, without any initiator addition, production of composites of $\mathrm{P}(\mathrm{AN}-\mathrm{co}-\mathrm{IA})$ with EDOT and MOT was performed via in-situ polymerization by addition of EDOT and MOT to the reaction medium at three different amounts for each monomer. Samples taken from the emulsion latexes were characterized by UV-visible spectroscopy, while the powder of the nanoparticles was analysed by FTIR-ATR spectroscopy and SEM. From the analysis results, it was observed that EDOT and MOT had successively polymerized in the structure. Beyond these characterizations, AFM characterization was also used for the spin-coated films obtained by treating the nanoparticles with DMF. By the help of this characterization, it can be clearly understood that the morphologies of the obtained particles are different from each other, and the morphology changes when the amount of monomers (EDOT and MOT) changes. After nanoparticle characterization, nanoparticles that had been treated with DMF and blended with PAN were electrospun. SEM results show that nanofibre diameters are found to be in the range of 94-180 $\mathrm{nm}$. EDS results proved that sulphur atoms in nanofibres belong to thiophene, which implies that blending of produced nanoparticles with PAN for electrospinning solution was well achieved. In addition, electrochemical measurements were carried out in order to study the electroactive behaviour of the electrospun nanofibres and compare the capacitive behaviour of the nanofibres with that of ITO-PET. In the light of these findings, it can be said that the obtained copolymers and nanofibres can be good candidates in many applications, including nanocoatings for textile industry, gas sensors, biosensors, etc., with further improvements where electroactivity is desired.

\section{Acknowledgment}

We thank Selin Gümrükçü for her contribution to the paper.

\section{References}

[1] Yanılmaz M, Kalaoğlu F, Karakas H and Sarac A S $2012 \mathrm{~J}$. Appl. Polym. Sci. 1254100

[2] Rahaman M S A, Ismail A F and Mustafa A 2007 Polym. Degrad. Stabil. 921421
[3] Bai Y-J, Wang C-G, Lun N, Wang Y-X, Yu M-J and Zhu B 2006 Carbon 441773

[4] Sen K, Hajir Bahrami S and Bajaj P 1996 J. Macromol. Sci. 361

[5] Han N, Zhang X-X and Wang X-C 2010 Iran. Polym. J. 19243

[6] Çetiner S, Sen S, Arman B and Sarac A S 2013 J. Appl. Polym. Sci. 1273830

[7] Bajaj P, Paliwal D and Gupta A K 1993 J. Appl. Polym. Sci. 49 823

[8] Han N, Zhang X-X, Wang X-C and Wang N 2010 Macromol. Res. 18144

[9] Yerlikaya Y, Unsal C and Sarac A S 2014 Adv. Sci. Eng. Med. 6301

[10] Unsal C, Kalaoğlu F, Karakas H, Sarac A S 2013 Adv. Polym. Technol. 32 E784

[11] Bhanu V A, Rangarajan P, Wiles K, Bortner M, Sankarpondian M, Godshall D et al 2002 Polymer 434841

[12] Devasia R, Reghunadhan Nair C P, Sadhana R, Babu N S and Ninan K N 2006 J. Appl. Polym. Sci. 1003055

[13] Sen S and Sarac A S 2014 J. Adv. Chem. 6958

[14] Arman B and Sarac A S 2014 Soft Nanosci. Lett. 442

[15] Devasia R, Reghunadhan Nair C P and Ninan K N 2002 Eur. Polym. J. 382003

[16] Yu M, Wang C, Zhao Y and Zhang M 2010 J. Appl. Polym. Sci. 1161207

[17] Bajaj P, Sreekumar T V and Sen K 2001 Polymer 421707

[18] Ouyang Q, Cheng L, Wang H and Li K 2008 Polym. Degrad. Stabil. 931415

[19] Tsai J S and Lin C H 1990 J. Mater. Sci. Lett. 9869

[20] Leclerc M and Faid K 1997 Adv. Mater. 91087

[21] Cheylan S 2006 Thin Solid Films 49716

[22] Ustamehmetoğlu B 2014 Electrochim. Acta 122130

[23] Tezcan C and Sarac A S 2010 J. Electrochem. Soc. 15799

[24] Satıc M T and Sarac A S 2015 Int. J. Polym. Mater. Polym. Biomater. 64597

[25] Fall M, Aaron J J, Sakmeche N, Dieng M M, Jouini M, Aeiyach S et al 1998 Synth. Met. 93175

[26] Fall M, Diagne A A, Dieng M M, Deflorian F, Rossi S, Bonora P L et al 2005 Synth. Met. 155569

[27] Sehgal P and Narula A K 2016 J. Solid State Chem. 233 428

[28] Huang Z-M, Zhang Y-Z, Kotaki M and Ramakrishna S 2003 Compos. Sci. Technol. 632223

[29] Doshi J and Reneker D H 1995 J. Electrostat. 35151

[30] Reneker D H and Yarin A L 2008 Polymer 492387

[31] Park E-S 2015 Polym. Compos. 361352

[32] Sulaiman Y and Kataky R 2012 Analyst 1372386

[33] Zhao Y, Wang C, Yu M, Cui C, Wang Q and Zhu B 2009 J. Polym. Res. 16437

[34] Tsegaye A A, Waryo T T, Admassie S and Iwuoha E I 2014 Int. J. Electrochem. Sci. 94840 